\title{
Chemistry with F Centers: Reduction of Organic Molecules on the Defective Potassium Chloride (100) Surface - SI
}

\author{
Michael Häfner, Manuel Hochheim, and Thomas Bredow* \\ Mulliken Center for Theoretical Chemistry, Institut für Physikalische und Theoretische \\ Chemie, Universität Bonn, Beringstraße 4, 53115 Bonn, Germany \\ E-mail: bredow@thch.uni-bonn.de
}

Phone: +49 (0)228 733839. Fax: +49 (0)228 739064 


\section{Electronic Property Calculations}

\section{Electronic Convergence Surface}

Table S1: CBM, VBM and $E_{g}$ of the pristine $\mathrm{KCl}(100)$ surface at varying amount of layers and saturation with ghost basis layers using sc-PBE0/adj-QVZPP in $\mathrm{eV}$.

\begin{tabular}{cccc}
\hline CBM & unsaturated & 1 ghost layer & 2 ghost layers \\
\hline 3 & 0.41 & -0.09 & -0.17 \\
4 & 0.33 & -0.07 & -0.16 \\
5 & 0.30 & -0.05 & -0.14 \\
6 & 0.28 & -0.04 & -0.13 \\
7 & 0.27 & -0.03 & -0.12 \\
\hline VBM & & & \\
\hline 3 & -8.88 & -8.89 & -8.89 \\
4 & -8.93 & -8.94 & -8.94 \\
5 & -8.92 & -8.93 & -8.93 \\
6 & -8.93 & -8.93 & -8.93 \\
7 & -8.92 & -8.93 & -8.93 \\
\hline$E_{g}$ & & & \\
\hline 3 & 9.29 & 8.81 & 8.72 \\
4 & 9.26 & 8.87 & 8.79 \\
5 & 9.22 & 8.88 & 8.79 \\
6 & 9.20 & 8.90 & 8.81 \\
7 & 9.19 & 8.90 & 8.81 \\
\hline
\end{tabular}




\section{Electronic Convergence Defective Surface}

Table S2: CBM and VBM for the $\alpha$-spin of the defective $\mathrm{KCl}^{*}(100)$ surface at varying amount of layers, supercell size and saturation with ghost basis layers using sc-PBE0/adj-QVZPP in eV.

\begin{tabular}{cccc}
\hline CBM & & & \\
supercell & unsaturated & 1 ghost layer & 2 ghost layers \\
\hline $3 \times 2 \times 2$ & 1.17 & 0.03 & -0.16 \\
$3 \times 3 \times 3$ & 0.72 & 0.02 & -0.12 \\
$3 \times 4 \times 4$ & 0.55 & -0.03 & -0.15 \\
$3 \times 5 \times 5$ & 0.48 & -0.06 & -0.16 \\
\hline $5 \times 2 \times 2$ & 0.65 & 0.09 & -0.10 \\
$5 \times 3 \times 3$ & 0.49 & 0.08 & -0.08 \\
$5 \times 4 \times 4$ & 0.40 & 0.00 & -0.11 \\
$5 \times 5 \times 5$ & 0.36 & -0.02 & -0.12 \\
\hline $7 \times 3 \times 3$ & 0.36 & 0.07 & -0.08 \\
$7 \times 4 \times 4$ & 0.32 & 0.01 & -0.10 \\
\hline $\mathrm{VBM}$ & & & \\
\hline $3 \times 2 \times 2$ & -2.78 & -3.30 & -3.30 \\
$3 \times 3 \times 3$ & -2.87 & -3.12 & -3.13 \\
$3 \times 4 \times 4$ & -2.88 & -3.08 & -3.09 \\
$3 \times 5 \times 5$ & -2.88 & -3.07 & -3.08 \\
\hline $5 \times 2 \times 2$ & -3.03 & -3.32 & -3.32 \\
$5 \times 3 \times 3$ & -3.15 & -3.34 & -3.34 \\
$5 \times 4 \times 4$ & -3.15 & -3.30 & -3.31 \\
$5 \times 5 \times 5$ & -3.16 & -3.29 & -3.29 \\
\hline $7 \times 3 \times 3$ & -3.16 & -3.35 & -3.35 \\
$7 \times 4 \times 4$ & -3.16 & -3.31 & -3.31 \\
\hline
\end{tabular}


Table S3: CBM, VBM and $E_{g}$ for the $\alpha$-spin of the $5 \times 4 \times 4$ model with two ghost basis layers of the defective $\mathrm{KCl}^{*}(100)$ surface for different shrinking factors of the Monkhorst-Pack grid using sc-PBE0/adj-QVZPP in eV.

\begin{tabular}{cccc}
\hline Grid & CBM & VBM & $E_{g}$ \\
\hline $1 \times 1 \times 1$ & \multicolumn{3}{c}{ SCF fails to converge } \\
$2 \times 2 \times 1$ & -0.11 & -3.31 & 3.20 \\
$4 \times 4 \times 1$ & -0.11 & -3.31 & 3.20 \\
$8 \times 8 \times 1$ & -0.11 & -3.31 & 3.20 \\
\hline
\end{tabular}

\section{Defective Surface}
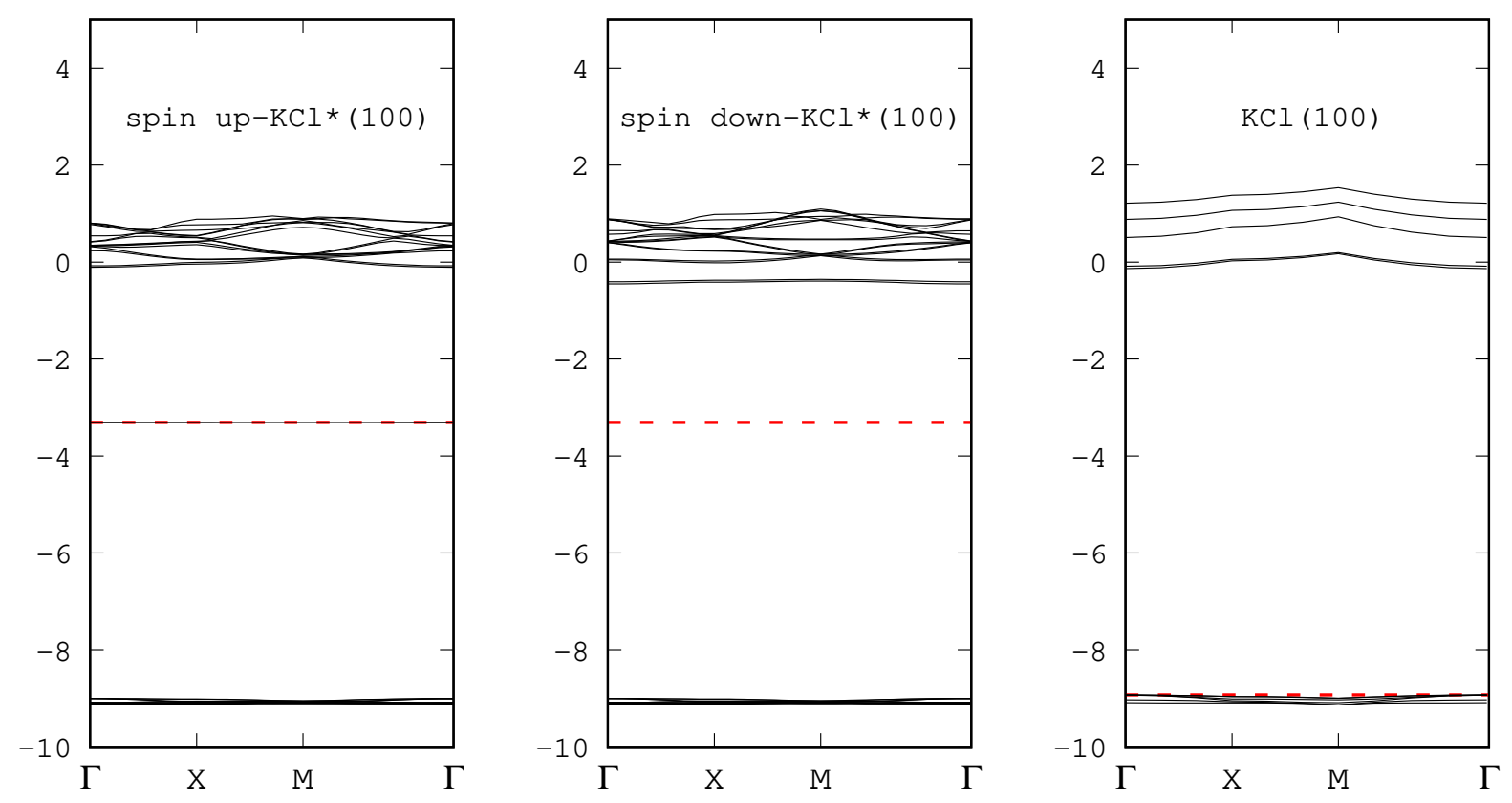

Figure S1: Band structure of $\mathrm{KCl}(100)$ and $\mathrm{KCl}^{*}(100)$. The red dashed line indicates the Fermi-level, energies are given in $\mathrm{eV}$. 


\section{Orbital Pictures of Selected Molecules}

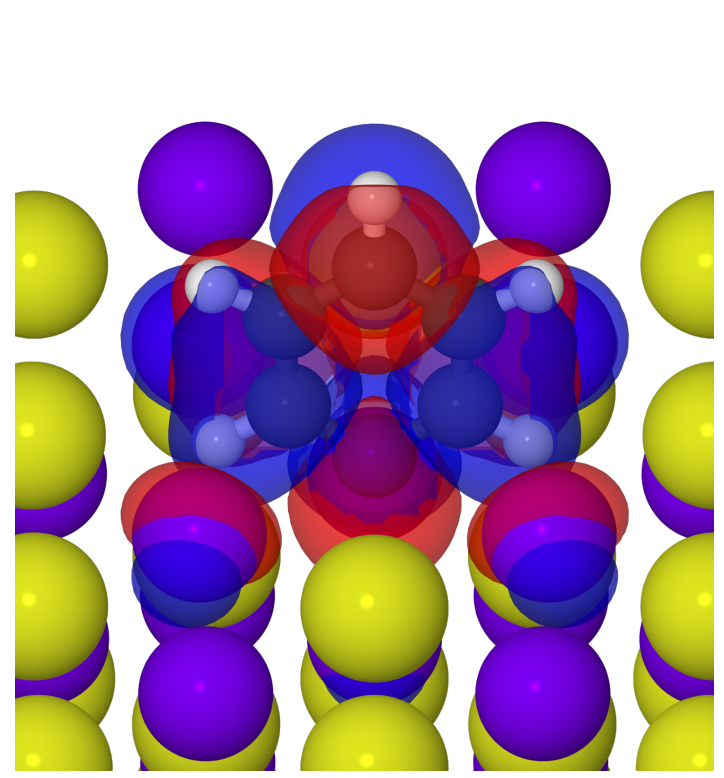

(a) SOCO of pyridine (12) on $\mathrm{KCl}^{*}(100)$.

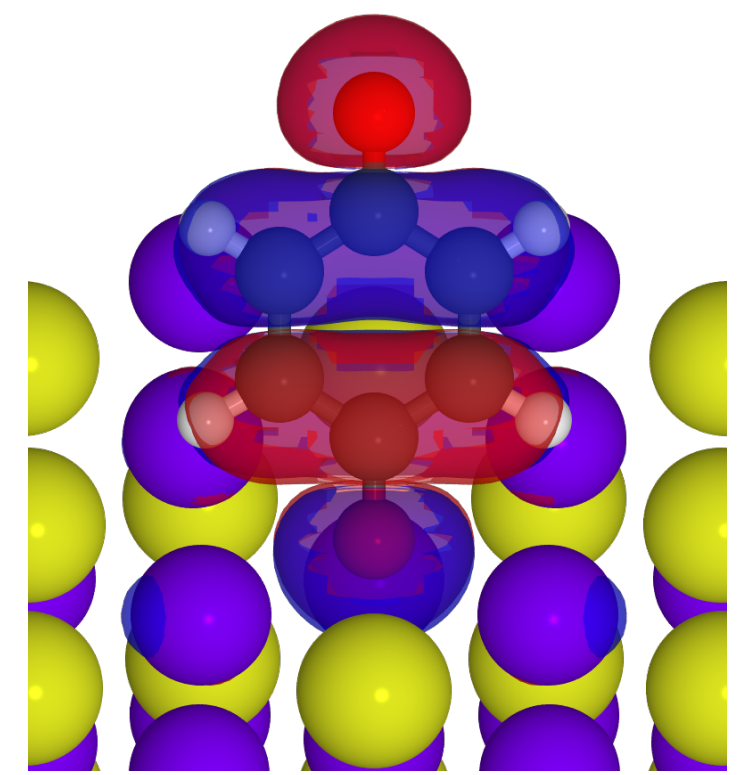

(b) SOCO of para-benzoquinone (10) on $\mathrm{KCl}^{*}(100)$.

Figure $\mathrm{S} 2$ : $\mathrm{SOCO}$ of $(\mathbf{1 0})$ and $(\mathbf{1 2})$ on $\mathrm{KCl}^{*}(100)$. In both cases, the $\mathrm{SOCO}$ is mainly equal to the LUMO of the free adsorbate. 


\section{Geometries of Remaining Molecules}

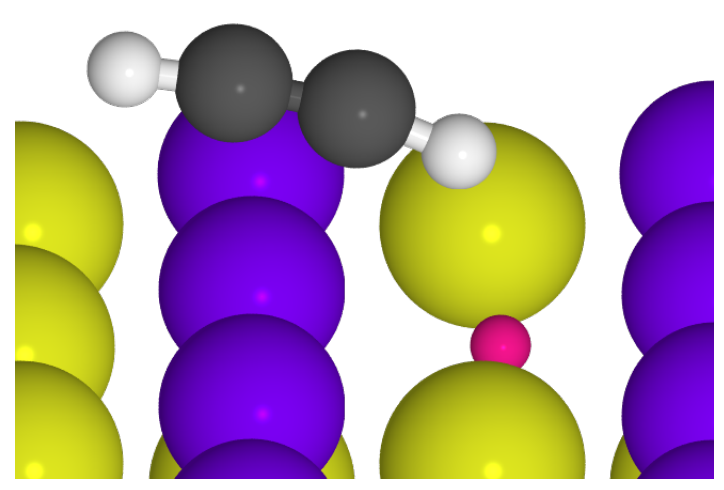

(a) Acetylene (1) on $\mathrm{KCl}^{*}(100)$.
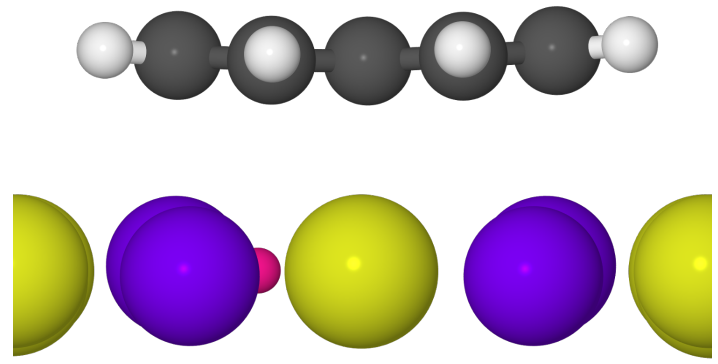

$-$

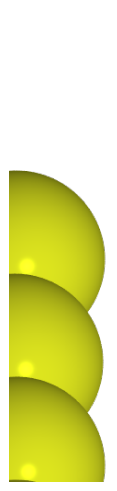

(b) Ethylene (2) on $\mathrm{KCl}^{*}(100)$.

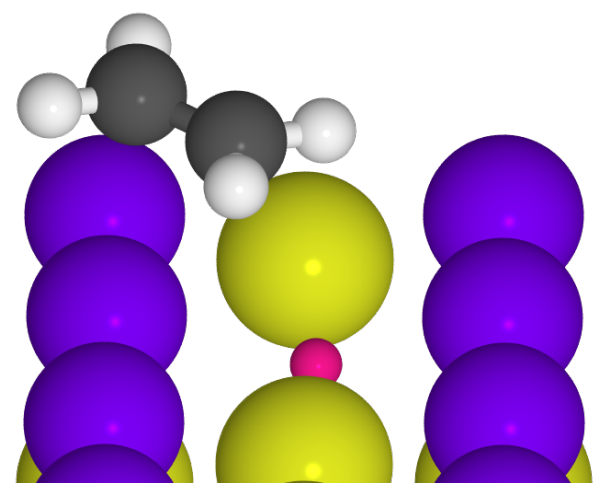

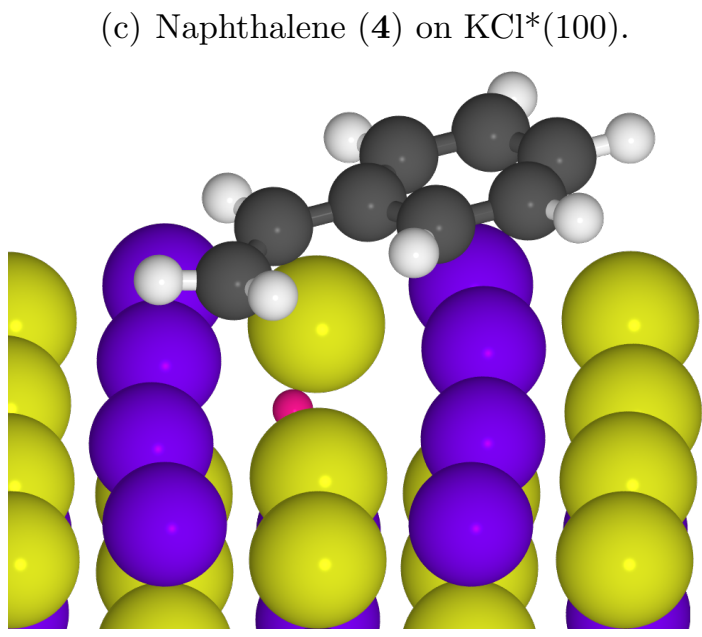

(e) Styrene (6) on $\mathrm{KCl}^{*}(100)$.
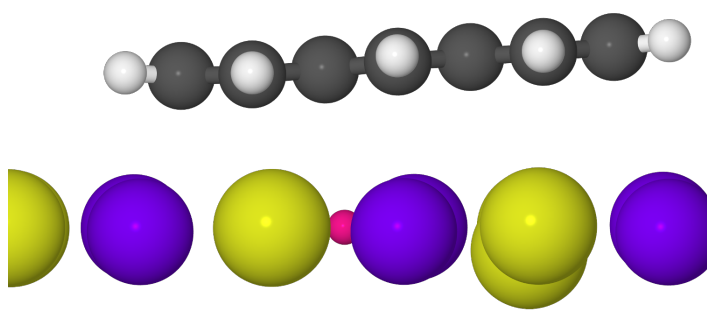

(d) Anthracene (5) on $\mathrm{KCl}^{*}(100)$.

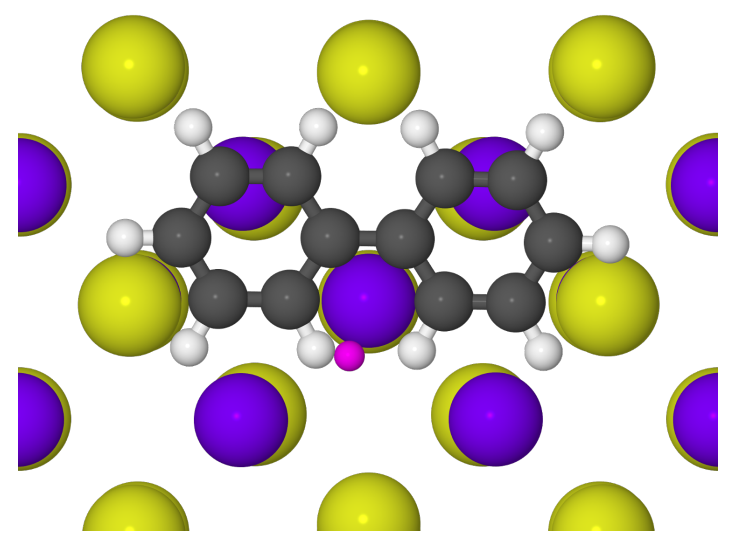

(f) Biphenyl (7) on $\mathrm{KCl}^{*}(100)$.

Figure S3: Final geometry of group 1 adsorbates on $\mathrm{KCl}^{*}(100)$ not shown before. The pink sphere is the location of the ghost atom describing the $\mathrm{F}_{\mathrm{S}}$ center. 


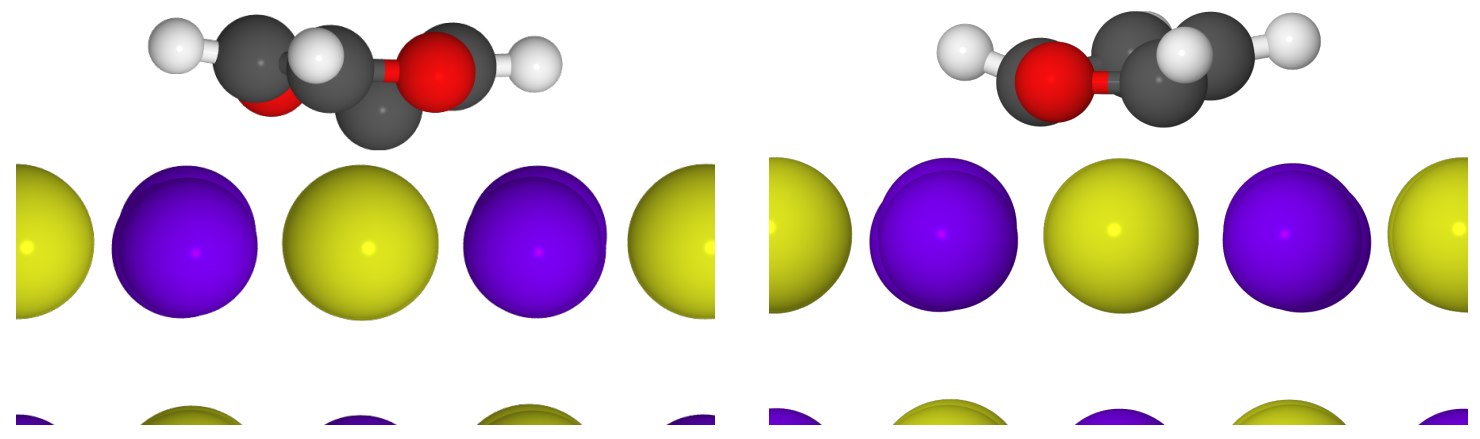

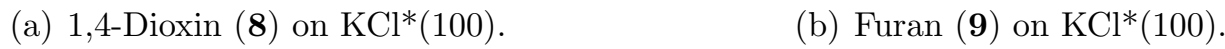

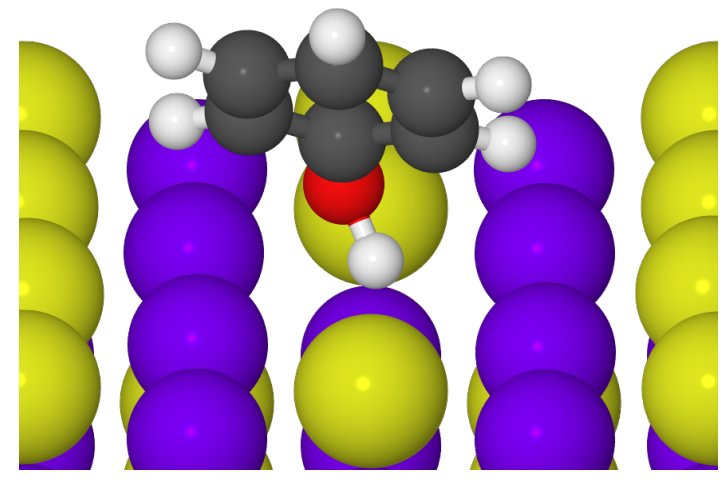

(c) Phenol (11) on $\mathrm{KCl}^{*}(100)$.

Figure S4: Final geometry of group 2 adsorbates on $\mathrm{KCl}^{*}(100)$ not shown before. 


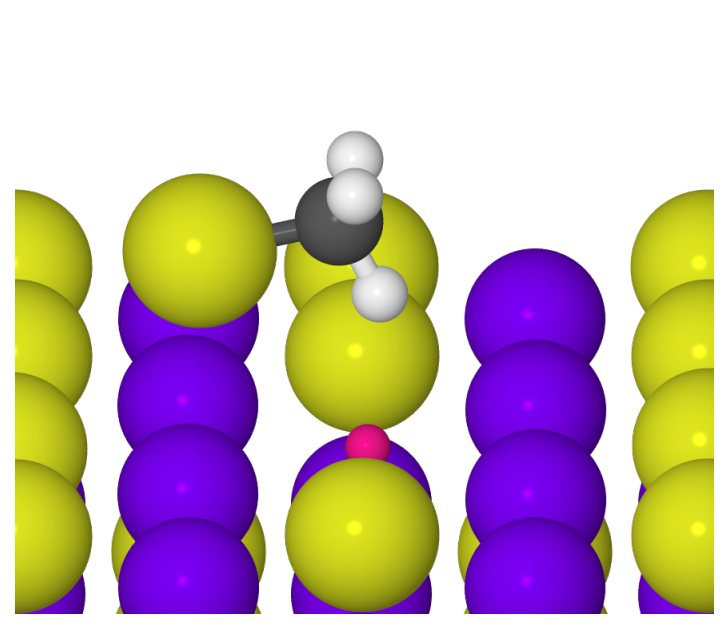

(a) Chloromethane (15) on $\mathrm{KCl}^{*}(100)$.

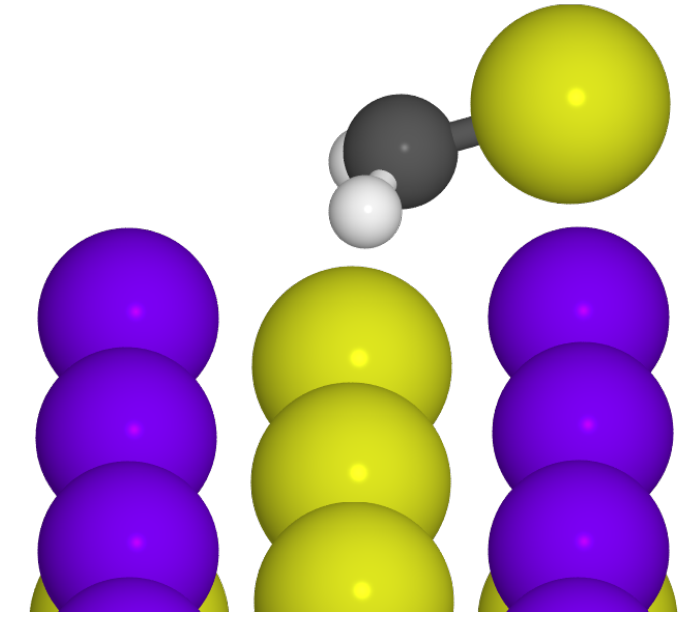

(b) Dissociated dichloromethane (16) on $\mathrm{KCl}^{*}(100)$.
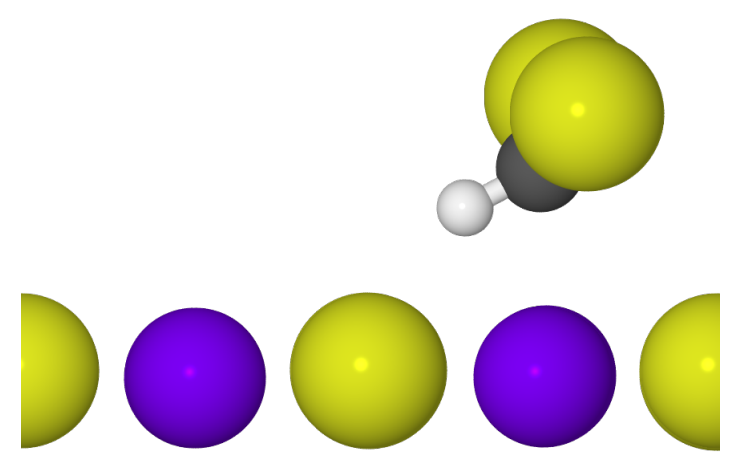

(c) Dissociated trichloromethane $\mathrm{KCl}^{*}(100)$.

Figure S5: Final geometry of some group 3 adsorbates on $\mathrm{KCl}^{*}(100)$. 


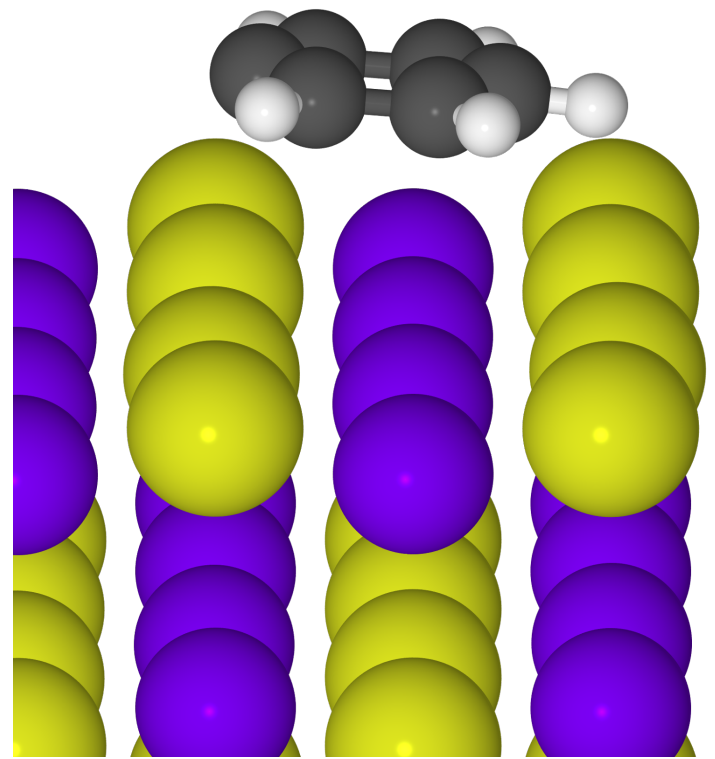

(a) Phenyl radical adsorbed to $\mathrm{KCl}(100)$ after chlorobenzene (19) was split by the defect.
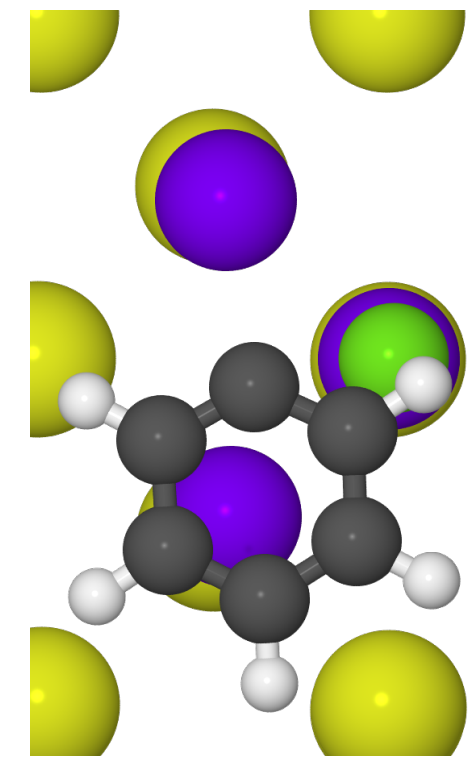

(c) Dissociated fluorobenzene $\mathrm{KCl}^{*}(100)$ the fluorine atom.

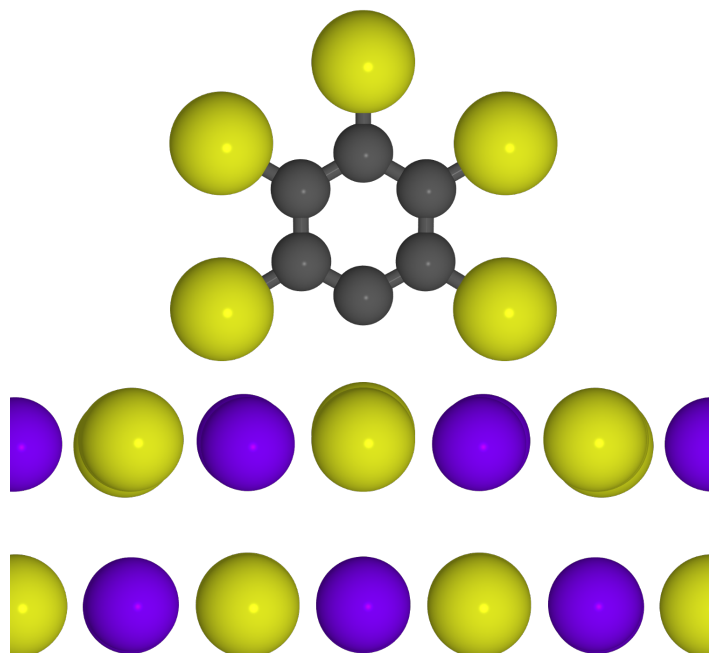

(b) Pentachlorophenyl radical adsorbed to $\mathrm{KCl}(100)$ after hexachlorobenzene (20) was split by the defect. Optimization with high symmetry, not necessarily most stable configuration.

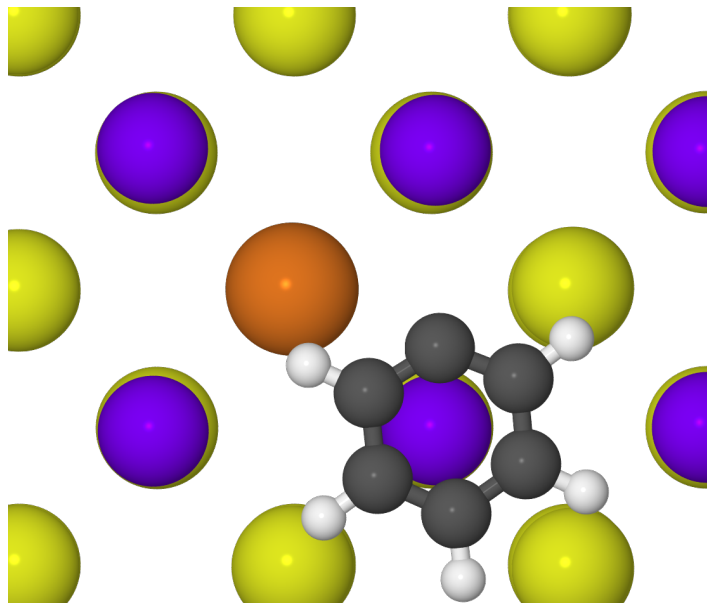

(d) Dissociated bromobenzene (22) on $\mathrm{KCl}^{*}(100)$. The orange sphere represents the bromine atom.

Figure S6: Final geometry of some group 3 adsorbates on $\mathrm{KCl}^{*}(100)$. 


\section{Adsorption energies of the halogenated radicals}

Table S4: Adsorption energies of the halogenated radical on the $\mathrm{KCl}(100)$ surface at $\mathrm{PBE} /$ pob-TZVP-rev2 level in $\mathrm{eV}$.

\begin{tabular}{ccccc}
\hline molecule & $\Delta E^{\mathrm{PBE}}$ & $\Delta E^{\mathrm{D} 3(\mathrm{BJ})}$ & $E^{\mathrm{cP}}$ & $\Delta E_{\text {tot }}$ \\
\hline $\mathrm{CH}_{3}$ & -0.26 & -0.12 & 0.18 & -0.20 \\
$\mathrm{CH}_{2} \mathrm{Cl} \bullet$ & -0.32 & -0.19 & 0.21 & -0.30 \\
$\mathrm{CHCl}_{2}^{\bullet}$ & -0.34 & -0.24 & 0.24 & -0.34 \\
$\mathrm{CCl}_{3}^{\bullet}$ & -0.39 & -0.36 & 0.38 & -0.36 \\
\hline
\end{tabular}

\section{Basis functions}

For accurate electronic properties, a large atom-centered basis set with diffuse functions is required and the pob-TZVP-rev2 basis set together with PBE as functional is insufficient. Especially for the description of anionic compounds diffuse basis functions are crucial. Because of this, we have performed a single-point calculation on each fully optimized geometry using an adopted basis set based on Ahlrich's def2-QVZPP ${ }^{1}$ and a self-consistent dielectricdependent global hybrid (sc-DDGH) functional based on PBE0 ${ }^{2}$. Without modification such large and diffuse basis sets cannot be used in CRYSTAL as they create pseudo linear dependencies. We circumvented this issue by cutting the most diffuse basis functions in the basis sets. The basis sets of hydrogen, nitrogen, oxygen, fluorine and bromine were used without any further modification. For chlorine, also the $f$-functions were removed. This improved computational performance without significantly changing the electronic properties. In addition, $g$-functions were removed as CRYSTAL can not handle functions with such high angular momentum.

Only the basis set for potassium and carbon had to be further modified by reducing the amount of diffuse basis functions. In the basis set of potassium, all functions with exponents of less than 0.07 and all $f$-functions were removed. The effect of the $f$-functions could be compensated with a single additional d-function with an exponent of 0.09 . The first change 
fixed the linear dependency issues for $\mathrm{KCl}$, the latter improved calculation performance significantly. It should be noted that most values of the exponents have been chosen based on empirical considerations, but they still yield variationally lower energies than a calculation with Ahlrich's def2-TZVPP basis set.

Construction of an adequate basis set for carbon is most difficult. The proposed adjusted QVZPP basis set (adj-QVZPP) was obtained variationally for diamond and augmented with a $p$-function with exponent 0.14. Just removing basis functions until the SCF converges yields results which are much worse than results obtained with a def2-TZVPP basis set.

\section{Addendum: Ghost functions}

In test calculations, we modeled the $\mathrm{F}_{\mathrm{S}}$ center using the chlorine basis functions and the diffuse $s$-functions. Both approaches yield electronic properties within the $0.1 \mathrm{eV}$ convergence criterion, indicating that diffuse $s$-functions alone are already sufficient for the defect electron description. To maintain consistency between the optimization and the electronic properties calculation, we continued to use the chlorine basis set to describe the vacancy.

Furthermore, we used the triplet configuration throughout this work as it retains a mirror symmetry and there is almost no difference to the singlet configuration.

\section{CRYSTAL integral tolerances}

A set of five parameters controls the accuracy of the calculation of bielectronic Coulomb and exchange series in CRYSTAL17 calculations ${ }^{3}$. They are given as a b c d e in the input and are evaluated as $10^{-\mathrm{a}}$ inside the calculation. Each parameter describes a different kind of overlap of two atomic orbitals corresponding to a bielectronic integral. Overlaps lower than this threshold are neglected. The thresholds had to be lowered in order to ensure sufficient accuracy. 


\section{References}

(1) Weigend, F.; Furche, F.; Ahlrichs, R. Gaussian Basis Sets of Quadruple Zeta Valence Quality for Atoms H-Kr. J. Chem. Phys. 2003, 119, 12753-12762.

(2) Perdew, J. P.; Ernzerhof, M.; Burke, K. Rationale for Mixing Exact Exchange with Density Functional Approximations. J. Chem. Phys. 1996, 105, 9982-9985.

(3) Dovesi, R.; Erba, A.; Orlando, R.; Zicovich-Wilson, C. M.; Civalleri, B.; Maschio, L.;

Rérat, M.; Casassa, S.; Baima, J.; Salustro, S. et al. Quantum-mechanical Condensed Matter Simulations with CRYSTAL. Wiley Interdiscip. Rev.: Comput. Mol. Sci. 2018, 8, No. e1360. 